\section{Políticas orientadas a misiones: ¿son posibles en Argentina?}

Resumen: En América Latina, a diferencia de los países centrales y los de industrialización reciente,las políticas de ciencia y tecnología (CyT) fueroninfluenciadas por una lógica basada en la oferta de conocimientos proveniente del sector académico. Esto se tradujo en la promoción sistémica de políticas horizontales: formación de recursos altamente calificados en CyT, creación de infraestructura CyT y financiamiento de proyectos de Investigación y Desarrollo $(I+D)$. La continuidad de esta tendencia derivó en importantes debilidades estatales para la definición e implementación de políticas focalizadas, centradas en sectores y tecnologías estratégicas, y en particular, de políticas orientadas a misiones enraizadas en problemas de relevancia socioeconómica. En este artículo, abordaremos los procesos que explican esta tendencia regional, así como dos estudios de caso de políticas orientadas a misiones (nuclear y de comunicación satelital)implementadas en el contexto argentino en los últimos años.

Palabras clave: políticas orientadas a misiones, proyectos tecnológicos estratégicos, Ciencia y tecnología en la semiperiferia, Ciencia y tecnología en América Latina

Mission-oriented policies: are they possible in Argentina?

Abstract: In Latin America, unlike the central countries and those of recent industrialization, science and technology (S\&T) policies were influenced by a logic based on the offer of knowledge coming from the academic sector. This was reflected in the systemic promotion of horizontal policies: training of highly qualified human resources, creation of S\&T infrastructure and financing of Research and Development (R\&D) projects. This trend produced important State weaknesses to impulse focused policies, centralized on strategic sectors and technologies, and mission-oriented policies rooted in problems of socioeconomic relevance. In this article, we will discuss this regional trend, as well as two case studies of mission oriented policies (nuclear and satellite communication) implemented in the Argentine context in recent years.

Keywords: mission oriented policies, technological strategic projects, S\&Tin the semiperiphery., S\&T in Latin America

Políticas orientadas a missões: são possíveis na Argentina?

Resumo: Na América Latina, diferentemente dos países centraes e recentes da industrialização,as políticas de ciência e tecnologia (C\&T) foraminfluenciadas por uma lógica acadêmicabaseada no suprimento de conhecimentodo setor académico. Issoresultounapromoçãosistêmica de políticas horizontais: treinamento de recursos altamente qualificadosem $C \& T$, criação de infraestruturaC\&T e financiamento de projetos de pesquisa e desenvolvimento (P\&D). Essatendênciaproduziu importantes fragilidades estatais para a definição de políticas focadasemsetores e tecnologias estratégicas, e da políticas orientadas amissões enraizadas em problemas de relevânciasocioeconômica. Neste artigo, discutiremos esta tendência regional, bem comodoisestudos de caso de políticas orientadas amissõesdesenvolvidas no contexto argentino nos últimos anos. Palavras chave: políticas orientadas a missões, projetos tecnológicos estratégicos, Ciência e tecnologia na semiperiferia, Ciência e tecnologiana América Latina

\section{Ciencia Tecnología y Política \\ Año 2 Nº jul- dic 2019}

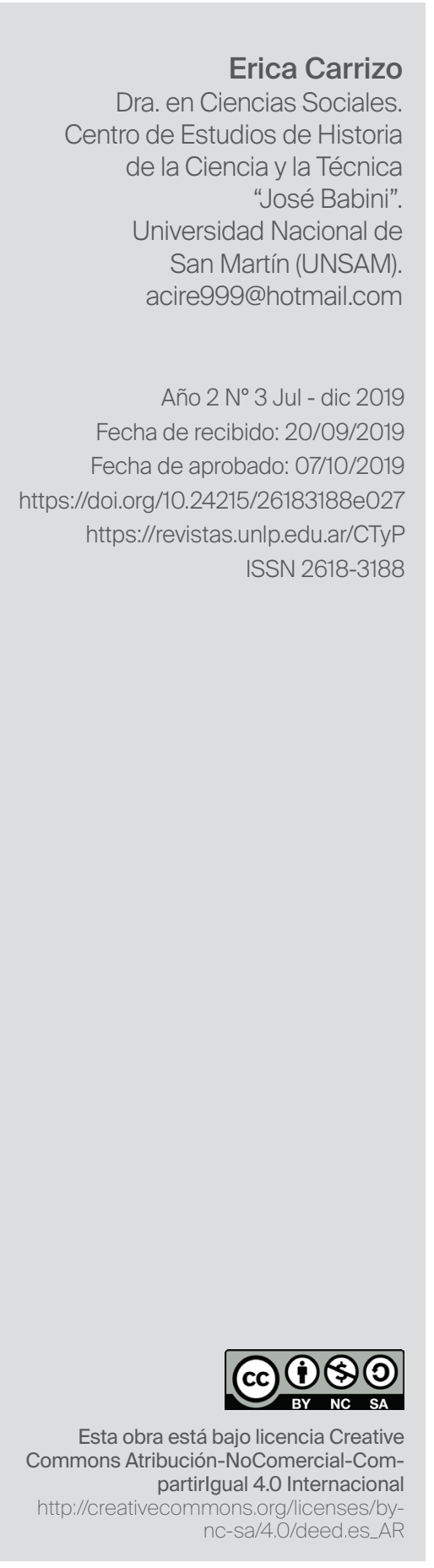

UNIVERSIDAD

NACIONAL

DE LA PLATA 
| Ciencia, Tecnología y Política | Año 2 | N³ | Julio-Diciembre 2019 | ISSN 2618-3188 | www.revistas.unlp.edu.ar/CTyP |

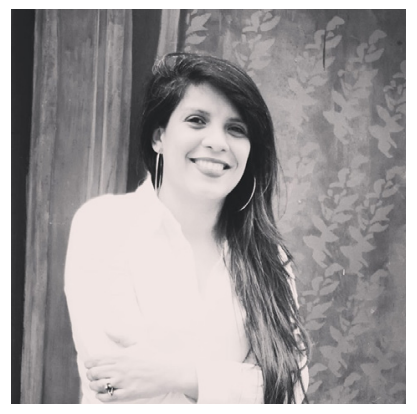

\section{Erica Carrizo}

Dra. en Ciencias Sociales.

Centro de Estudios de

Historia de la Ciencia y la

Técnica “José Babini”.

Universidad Nacional de

San Martín (UNSAM).

acire999@hotmail.com

\section{Políticas orientadas a misiones: ¿son posibles en la Argentina?}

Resumen: En América Latina, a diferencia de los países centrales y los de industrialización reciente,las políticas de ciencia y tecnología (CyT) fueroninfluenciadas por una lógica basada en la oferta de conocimientos proveniente del sector académico. Esto se tradujo en la promoción sistémica de políticas horizontales: formación de recursos altamente calificados en CyT, creación de infraestructura CуT y financiamiento de proyectos de Investigación y Desarrollo (I+D). La continuidad de esta tendencia derivó en importantes debilidades estatales para la definición e implementación de políticas focalizadas, centradas en sectores y tecnologías estratégicas, y en particular, de políticas orientadas a misiones enraizadas en problemas de relevancia socioeconómica. En este artículo, abordaremos los procesos que explican esta tendencia regional, así como dos estudios de caso de políticas orientadas a misiones (nuclear y de comunicación satelital)implementadas en el contexto argentino en los últimos años.

\section{Políticas de ciencia, tecnología e innova- ción en países semiperiféricos}

En los años que siguieron a la Segunda Guerra Mundial (1939-1945), la ciencia y la tecnología (CyT) pasaron a ser, a nivel global, un tema estratégico para los Estados. Sobre la base de perspectivas contrapuestas sintetizadas en las posiciones enfrentadas de John D. Bernal (1939) y Michael Polanyi (1951) - planificación del Estado versus libertad de investigación y autonomía científica-, la política de CyT mundial, que a partir de la década de 1980 comienza a ser mayormente referenciada como política de ciencia, tecnología e innovación (CTI), sufrió un importante cambio. Evolucionó desde iniciativas horizontales dirigidas a fortalecer la infraestructura y las capacidades científico-tecnológicas de los países, pasando por iniciativas focalizadas en sectores y tecnologías de interés especial, hasta derivar en políticas orientadas a misiones, dirigidas a objetivos específicos. Estas políticas, comúnmente denominadas en inglés mission oriented policies (MOP) o challenge policies, se caracterizan por im- 
pulsar grandes proyectos tecnológicos estratégicos orientados por un objetivo. Se trata de políticas sistémicas que coordinan y ejecutan una gran diversidad de instrumentos financieros y no financieros a través de los cuales el Estado vincula actores públicos y privados para el desarrollo de sectores, tecnologías y mercados según objetivos estratégicos propios (Ergas, 1987, Peres y Primi, 2009; Mazzucato, 2014 [2013]; Mazzucato, 2014; Mazzucato y Penna, 2016), en las que el punto de partida y de llegada son las necesidades sociales (Boon y Edler, 2018: 436). Estas políticas orientadas a misiones deben ser entendidas como intentos holísticos de articular diferentes políticas, mediante una variedad de tecnologías y dispositivos sociotécnicos, orientados a resolver desafíos definidos colectivamente a través de procesos sociales, o más frecuentemente, a través de procesos políticos (Boon y Edler, 2018: 445).

Sin embargo, las posibilidades de un país para definir e implementar estas políticas no son independientes del contexto donde opera -centro, semiperiferia o periferia- como tampoco de las capacidades y las lógicas que en éste subyacen al diseño y la gestión de los vínculos entre ciencia-tecnología-industria y desarrollo socioeconómico.

Siguiendo la definición de Immanuel Wallerstein (1974), en la estructura económica mundial pueden identificarse tres zonas: i) el centro: conformado por países que se apropian desproporcionadamente de los beneficios de la división internacional del trabajo y dominan los sectores intensivos en conocimiento; ii) la periferia: conformada por países con economías primarias basadas en la producción de materias primas; y iii) la semiperiferia: conformada por países con cierta capacidad industrial y tecnológica que pretenden competir en algunos segmentos del mercado mundial definidos por las aplicaciones comerciales basadas en tecnología.

Las economías centrales y las de industrialización reciente rápidamente concentraron sus esfuerzos en MOP, guiadas por una lógica burocrática preocupada por aplicar la tecnología a la resolución de problemas prácticos en sectores socioeconómicamente estratégicos y centralizada en la acción estatal. En América Latina, en cambio, a excepción de ciertos "bolsones de eficiencia" (Evans, 1995: 64-65) como fueron el sector aeronáutico en Brasil y el nuclear en Argentina, primó una lógica académica basada en la oferta de conocimientos, que alimentó la promoción sistémica de políticas horizontales. La continuidad de esta tendencia hasta la actualidad derivaría en importantes debilidades estatales para la definición e implementación de políticas focalizadas, y en particular de MOP enraizadas en problemas de relevancia socioeconómica para la región.

En el contexto latinoamericano, estas falencias son consecuencia del divorcio histórico entre la producción académica y la realidad socioeconómica, que incluye a los problemas de la producción. A diferencia de los países centrales y los países de industrialización reciente que supieron vincular sus actividades de Investigación y Desarrollo (I+D) con sectores estratégicos para su desarrollo socioeconómico ${ }^{1}$ en países como Argentina, Brasil o México, que presentan ciertas capacidades industriales y tecnológicas, los procesos de "desarrollo dependiente" (Evans, 1979), que desdibujan el rol del Estado en la producción de conocimiento, y sus ciclos recurrentes de inestabilidad política, económica y social, explican en buena medida la "desconexión" de las actividades de CyT con las realidades socioeco-

\footnotetext{
1 Ver, entre otros, Ergas 1987; Okimoto, 1989; Nelson, 1990; Amsden, 2001; Chiang, 1991; Evans 1995; Hecht 2001; Wade (2003 [1990]); Di Maio, 2009.
} 
nómicas locales.

\section{Características de las políticas orientadas a misiones: institucionalidad y transversalidad}

Si bien son muy disímiles las experiencias de las naciones modernas, tanto las de desarrollo temprano como tardío ${ }^{2}$, en las formas a través de las cuales el Estado intervino en la vinculación entre ciencia-tecnología-industria y desarrollo socioeconómico, y en la aplicación de MOP, es posible identificar algunos puntos de convergencia.

Entre estos puntos destacan el sector y las tecnologías estratégicas en las que se deciden focalizar los esfuerzos. Sin embargo, la selección y la apuesta por un sector estratégico y, en este marco, por las tecnologías estratégicas que consolidarán su desarrollo y sustentabilidad a largo plazo no es algo que pueda tomarse prestado. Esto debe ser el resultado de decisiones estratégicas por parte del Estado, cuyo punto de partida será, precisamente, su posición en la división internacional del trabajo y las posibilidades de avance que represente su capacidad de gestión del desarrollo tecnológico de su industria nacional (Carrizo, 2019: 66).

Ahora bien, un sector económica o industrialmente estratégico refiere a un nicho particular dentro de la economía de una nación que ofrece posibilidades contingentes de transformación industrial y, en consecuencia, de insertar productos o tecnologías de modo exitoso en cadenas de valor globales. Los sectores estratégicos, dan lugar a industrias económicamente estratégicas, es decir, industrias intensivas en I+D con efectos multiplicadores en el resto de la economía con costos decrecientes de producción. Las mismas se caracterizan por una competencia imperfecta, y en consecuencia, habitualmente suelen ser foco de la política comercial estratégica del Estado que le permite obtener una parte de los beneficios generados por estas industrias en los mercados mundiales (Hartley, 2012: 187). Las industrias estratégicas se basan en el uso, mejora y difusión de tecnologías estratégicas, las cuales se definen como tecnologías con capacidad de alimentar el desarrollo y la evolución de los "ecosistemas de innovación" o los "ecosistemas de aprendizaje, escalamiento e innovación", según hablemos de los países de la zona central o semiperiférica, como así también de posibilitar ganancias monopólicas u oligopólicas en los mercados internacionales para los gobiernos que las financien y protejan.

En un contexto semiperiférico, como el argentino, es importante destacar la relevancia que tienen estos ecosistemas de aprendizaje, escalamiento e innovación para el desarrollo de industrias económicamente estratégicas como así también para la implementación de MOP. Ahora bien, la palabra "ecosistema" proviene de la biología, en especial de la biología sistémica, mientras que el término "sistema" alude a un todo compuesto por varias partes o miembros que interactúan entre sí y actualmente se utiliza para describir a un set de componentes interdependientes interactuando de forma integrada. En los países centrales es común hablar de "ecosistema de innovación o emprendedor" (Isemberg, 2011; The World Economic Forum, 2013; Mazzucato, 2014 [2013]; Schwarzkopf, 2016 [2015]) para referenciar a un sistema integrado por una serie de componentes que incluyen dimensiones políticas, financieras, culturales, institucionales, de infraestructura, regulatorias, comerciales, científico-tecnológicas, educativas y de entrenamiento, entre otros, así como también dinámicas de interacción y aspectos conductuales.

Sin embargo, en el contexto de los países semiperiféricos no hay "sistemas" y la innovación es escasa.

${ }^{2}$ El análisis presentado en esta sección toma como base la experiencia de EEUU, como un ejemplo de desarrollo temprano, y las de Japón, Corea del Sur, y Taiwán, como casos de desarrollo tardío o industrialización reciente. 
En cambio se observan experiencias fragmentarias de desarrollo científico-tecnológico-industrial que logran generar ecosistemas densos, caracterizados por largos, pacientes e idiosincráticos procesos de aprendizaje, acumulación de capacidades tecnológicas y gerenciales basados en la copia y el escalamiento de mejoras científico-tecnológicas incrementales. Por esta razón, en este artículo utilizaremos en estos casos el concepto de "ecosistema de aprendizaje, escalamiento e innovación". Consideramos a este concepto como una extensión del de "ecosistema de innovación o emprendedor", pero que incluye las características propias de los procesos de desarrollo CTI en un contexto semiperiférico (Carrizo, 2019: 26).

Sin embargo, un país no sólo debe atender a cuestiones de índole económica o industrial, sino que su Estado también debe actuar seleccionando sectores socialmente estratégicos, cuyas lógicas no responden a las del mercado, ya que se vinculan a la generación de bienes y servicios de interés público -educación, salud, vivienda, alimentación, medioambiente, transporte, etc-. Las tecnologías estratégicas en este marco asumen tal carácter, no por alimentar a los ecosistemas de innovación o de aprendizaje, escalamiento e innovación, sino por su capacidad para resolver problemas socialmente relevantes.

El último eslabón que completa este esquema, sin dudas, el de mayor complejidad, es precisamente el de los problemas relevantes cuyo contenido debe definirse en función de las principales necesidades que un país deba resolver en un contexto sociohistórico dado, guiándose por la consigna del bienestar de las mayorías. La capacidad del Estado para identificar y trabajar en torno a estos problemas que no reconocen fronteras ya que son de naturaleza trans-sectorial, trans-territorial, y por sobre todo, trans-científica-tecnológica, es altamente dependiente del grado de enraizamiento socioeconómico del proyecto nacional vigente, y de cómo el país percibe y gestiona su inserción en el sistema capitalista global.

Finalmente, cabe aclarar que la estrategia que cada país adopta en una particular coyuntura sociohistórica -y que incluye al conocimiento útil 3; las capacidades estatales; los sectores, tecnologías e industrias estratégicas; los ecosistemas de innovación o de aprendizaje, escalamiento e innovación; y los problemas relevantes-, sólo adquiere sentido hacia el interior de las fronteras nacionales. Ninguno de estos engranajes significa nada si se lo analiza y define de modo aislado.

No obstante, otras dos dimensiones claves a la hora de analizar las condiciones de posibilidad de las MOP son la transversalidad y la institucionalidad. En los casos de la experiencia internacional estudiados, se observó que la transversalidad se corporizó en las estrechas vinculaciones que se establecen entre las políticas públicas del campo científico, tecnológico, educativo, comercial y financiero. Esquema en el que la política tecnológica se configuró como el núcleo organizador de la política de CTI, y la política industrial como el motor del desarrollo socioeconómico. Esta última política se asocia a una serie de medidas de diversos ámbitos de competencia de la acción pública y comprende a las políticas que impactan en las industrias estratégicas en desarrollo, como son las políticas macroeconómicas, las comerciales, las de CTI, las de compra pública, las de regulación de la inversión extranjera, las de propiedad intelectual, y las de direccionamiento de

\footnotetext{
${ }^{3}$ Tomamos como referencia la noción de "conocimiento útil" (Mokyr, 2005) para referirnos a todo aporte del campo del conocimiento que con criterios sociales convencionales se considera que contribuye a la resolución de un problema práctico (de tipo técnico o tecnológico, pero también organizativo, institucional, social, económico, político, etc.). Esto incluye tanto a los conocimientos científicos, de carácter descriptivo o propositivo, como así también a los de base no científica, como los conocimientos técnicos o artesanales.
} 
las fuentes de financiamiento (Cimoli, Dosi y Stiglitz, 2009: 2).

La otra dimensión, la institucionalidad, refiere a que las MOP generalmente son centralizadas por instituciones estatales que juegan un papel protagónico en la política industrial. En EEUU esta institución fue la Agencia de Proyectos de Investigación Avanzada en Defensa (DARPA), mientras que en Japón fue el Ministerio de Comercio Internacional e Industria (MITI), en Corea del Sur el Centro de Planificación Económica (CPE), y en Taiwán, el Consejo de Planificación Económica y Desarrollo (CEPD).

\section{Dos casos de políticas orientadas a misio- nes en Argentina}

Si bien la Argentina, como el resto de América Latina, históricamente ha tenido problemas para vincular su desarrollo CTI con su realidad socioeconómica, así como para definir una política de CTI sistémica, existen ciertas iniciativas que podemos asociar a la definición de MOP. Una de ellas estuvo centrada en el sector nuclear, un sector estratégico que el país viene impulsando desde la segunda mitad del siglo XX. La otra en el sector de comunicación satelital, que se configura como un desprendimiento del anterior. Este sector inicia su trayectoria en la década de 1990 a partir de la fabricación de satélites de observación y adquiere un acelerado proceso de desarrollo incremental durante los primeros años de la década del 2000.

Ambos casos configuran proyectos tecnológicos estratégicos basados en tecnologías estratégicas que son el resultado de largos, pacientes e incrementales procesos de aprendizaje tecnológico. En términos institucionales, estos proyectos como sus políticas públicas asociadas estuvieron coordinados por el hoy extinto Ministerio de Planificación Federal, Inversión Pública y Servicios (MINPLAN) en el período 2007-2015.
En el sector nuclear, el caso MOP que focalizaremos es el proyecto CAREM, que aunque tuvo sus orígenes en la década de 1980 en la Comisión Nacional de Energía Atómica (CNEA), después de un largo proceso de parálisis, fue reactivado en 2006 en el marco del relanzamiento del Plan Nuclear Argentino durante el gobierno de Néstor Kirchner (2003-2007). El mismo contempla el desarrollo y diseño de una planta nuclear de baja potencia. El problema socioeconómico que orientó este proyecto fue abastecer de electricidad a pequeños polos fabriles y a ciudades alejadas de los grandes centros urbanos. Si la Argentina lograra finalizar este reactor, hoy paralizado por las políticas del gobierno de Mauricio Macri, podría posicionarse a la vanguardia del mercado de plantas de baja potencia de última generación. Nos convertiríamos así en uno de los líderes mundiales en la fabricación de reactores modulares de baja y media potencia, junto a EEUU, Canadá, Rusia y China.

El otro ejemplo de una MOP es el de los satélites geoestacionarios ARSAT 1 y 2, lanzados en 2014 y 2015 respectivamente, los cuales fueron posibles gracias a la creación de la empresa estatal ARSAT en 2006. Recordemos que como parte del Plan Geoestacionario Argentino 2015-2035 estaba planificado fabricar el ARSAT 3, pero este proyecto fue abortado a inicios del gobierno de Mauricio Macri. En el caso de los satélites que sí alcanzaron a fabricarse, el problema socioeconómico que orientó estos proyectos fue abastecer de servicios de comunicación satelital a la Argentina y otros países del continente americano, lo cual posibilitó comercializar con tecnología nacional el acceso a servicios de internet, telefonía y TV digital abierta. Por otro lado, en los inicios de la trayectoria de la empresa ARSAT, la fabricación de estos satélites le permitió a la Argentina ingresar al grupo de los diez países del mundo -junto a EEUU, Rusia, China, India, Japón, Alemania, Francia, Italia e Israel- y ser el único del hemisferio sur, con capaci- 
dad de desarrollar satélites geoestacionarios de telecomunicaciones, una de las industrias más competitivas a escala global.

Ahora bien, la trayectoria evolutiva de los sectores nuclear y de comunicación satelital, en el marco de la cual se desarrollaron estas políticas, muestra una conjunción de variables en el plano geopolítico, geoeconómico, científico-tecnológico-industrial e institucional, que reflejan las limitaciones y oportunidades que implica, para un país de la semiperiferia, la decisión de impulsar procesos de desarrollo tecnológico autónomo en industrias económicamente estratégicas basadas en el conocimiento y globalmente competitivas. La principal lección que nos deja el estudio de estos dos casos es que la posibilidad de impulsar proyectos tecnológicos estratégicos con capacidad de impacto socioeconómico no es independiente del grado de autonomía con el que cuenta el Estado para orientar, por un lado, las capacidades y el conocimiento intangible instalados en el país. Y por otro lado, la conformación de entornos institucionales favorables al desarrollo de procesos de aprendizaje acumulativo que se propongan el crecimiento, la diversificación y el enraizamiento de trayectorias sectoriales en un entorno sistémico (Carrizo, 2019: 303).

\section{Conclusiones}

La orientación de la CTI en Latinoamérica históricamente se ha caracterizado por la definición de políticas horizontales, influenciadas por la lógica académica, basada en la oferta de conocimientos, y por procesos de desarrollo dependiente que coinciden con períodos de gobierno de orientación neoliberal, que han reforzado y consolidado esta característica.

Contrariamente, tanto en los países centrales como en los de industrialización reciente -EEUU, Japón, Corea del Sur y Taiwán-, se observó el direccionamiento de los esfuerzos hacia políticas focalizadas y
MOP, para las cuales se requieren priorizar sectores, tecnologías y problemas estratégicos.

Del análisis de estas experiencias internacionales surgen algunos aspectos comunes a tener en cuenta que parecerían caracterizar estas políticas. Por un lado, la institucionalidad, dado que la gestión de estas MOP habitualmente se concentra en instituciones estatales orientadas según objetivos específicos. Por el otro, la transversalidad, ya que en estas experiencias fue posible observar una estrecha vinculación entre las políticas de CTI, educativa, comercial, financiera e industrial.

Sin embargo, en la Argentina, las iniciativas que podrían clasificarse como MOP, como el proyecto CAREM y los satélites geoestacionarios ARSAT 1 y 2, no estuvieron asociadas a una política de CTI ni industrial centralizada, ni sistémica, sino que emergieron en el marco de sectores económicamente estratégicos cuyos antecedentes se remontan a los inicios del sector nuclear en la década de 1950.

En estos casos, el ecosistema de aprendizaje, escalamiento e innovación nuclear-espacial argentino se configuró como una dimensión clave para el impulso y desarrollo, en el contexto nacional, de estas MOP.

En consecuencia, y sin intenciones de motivar visiones generalizadoras, podríamos decir que la posibilidad de diseñar y gestionar iniciativas de este tipo debe tener en cuenta aspectos de índole geopolítico y geoeconómico. En particular el rol de las fronteras nacionales, y la estrategia que cada país define para enfrentar los problemas relevantes en los que decide focalizar sus esfuerzos.

Ahora bien, dado el peso cultural del paradigma eurocéntrico y su amplificación a través de la acción proselitista de los organismos internacionales de crédito en el ámbito de la política de CTI latinoamericana, existe una tendencia a la adscripción acrítica a recetas, manuales de procedimientos y metodolo- 
gías de supuesto alcance universal para resolver los problemas que plantea el desarrollo CTI en las sociedades modernas. Fuente inagotable de estudios de consultoría y contratos de asesoría mercenaria que -icasualmente?- recomiendan las mismas prescripciones para todos los países que integran el bloque del "subdesarrollo".

Los resultados de este enfoque están a la vista: América Latina todavía sigue esperando el milagro y la aparición, repentina, inevitable y necesaria, del nuevo "gurú" que le indique la salida. Sin embargo, esta autora es enfática en remarcar la importancia de desarticular la falacia imitativa, según la cual los problemas del desarrollo científico-tecnológico-industrial latinoamericano se resuelven copiando -y pagando- a un "otro", siempre más avanzado, moderno, evolucionado. Esta visión reduccionista no reconoce a los senderos de desarrollo socioeconómico, científico, tecnológico e industrial como construcciones sociohistóricas inimitables, y condicionadas por la inserción y evolución de nuestros países en el juego geopolítico y geoeconómico global; las características de la estructura productiva, del complejo científico-tecnológico y de la esfera social nacional; y la puja entre los modelos de país vigentes en cada coyuntura sociohistórica.

\section{Bibliografía}

Amsden, A. (2001) The Rise of 'The Rest': Challenges to the West from Late-Industrializing Economies. Oxford: Oxford University Press.

Bernal, J. (1939) The Social Function of Science. Londres: George Routledge \& Sons.

Boon, W. y Edler, J. (2018) "Demand, challenges, and innovation. Making sense of new trends in innovation policy", Science and Public Policy, 45/ 4: 435-447.

Carrizo, E. (2019) Las políticas de ciencia, tecno- logía e innovación en contexto semiperiférico: el caso argentino en el período 2007-2017. Tesis doctoral. Universidad de Buenos Aires (UBA). Buenos Aires. Argentina.

Cimoli, M., Dosi, G. y Stiglitz, J. (2009) The Political Economy of Capabilities Accumulation: The Past and Future of Policies for Industrial Development. En M. Cimoli, G. Dosi y J. Stiglitz (Eds.), Industrial Policy and Development. The Political Economy of Capabilities Accumulation (pp. 1-16.). Oxford: Oxford University Press.

Di Maio, M. (2009) "Industrial Policies in Developing Countries: History and Perspectives". In Cimoli, M., Dosi, G., and Stiglitz, J. (Eds.) Industrial Policy and Development. The Political Economy of Capabilities Accumulation, pp. 107-143. Oxford: Oxford University Press.

Ergas, H. (1987) Does Technology Policy Matter? En B. Guille y H. Brooks (Eds.), Technology and Global Industry: Companies and Nations in the World Economy (pp. 191-280). Washington DC: National Academy Press.

Evans, P. (1979) Dependent Development. The Alliance of Multinational, State, and Local Capital in Brazil. New Jersey: Princeton University Press.

-- (1995) Embedded Autonomy: States and Industrial Transformation. Princeton: Princeton University Press.

Hartley, H. (2012) The economic of defense policy. A new perspective. UK: University of York.

Herrera, A. (1995[1975]) "Los determinantes sociales de la política científica en América Latina: Política científica explicita y Política científica implícita", Redes, 2/ 5: 117-131.

Hecht, G. (2001). Technology, Politics, and Nacional Identify in France. In M. Allen y G. Hecht (Eds.) Technologies of Power. Cambrige: The MIT Press. 
Isenberg, D. (2011) "Introducing the Entrepreneurship Ecosystem: Four Defining Characteristics", Forbes Magazin.

Mazzucato, M. (2014[2013]) El Estado emprendedor. Mitos del sector público frente al sector privado. Barcelona: RBA Libros.

-- (2014). A mission-oriented approach to building the entrepreneurial state. A 'think piece' for the Innovative UK. Obtenido el 16 de noviembre de 2017 de http://marianamazzucato.com/ wp-content/uploads/2014/11/MAZZUCATO-IN NOVATE-UK.pdf.

-- y Penna, C. (2016) The Brasilian Innovation System: A Mission-Oriented Policy Proposal. Brasilia: Centro de Gestão e Estudos Estratégicos (CGEE). Mokyr, J. (2005) Long-Term Economic Growth and the History of Technology. En Ph. Aghion y S. N. Durlauf (Eds.), Handbook of Economic Growth (pp. 1113-1180). The Netherelands: Elseiver.

Nelson, R. (1990). "U.S. technological leadership: Where did it come from and where did it go?", Research Policy, 19/2: 117-132.

Okimoto, D. (1989) Between MITI and the Market: Japanese Industrial Policy for High Technology. Stanford: Stanford University Press.

Peres, W. y Primi, A. (2009) Theory and practice of industrial policy. Evidence from the Latin American Experience. Santiago de Chile: United Nations.

Polanyi, M. (1951) The Logic of Liberty. Londres: Routledge \& Kegan Paul.

Schawarzkopf, C. (2016[2015]) Fostering Innovation and Entrepreneurship. Entrepreneurial Ecosystem and Entrepreneurial Fundamentals in the USA and Germany. Wiesbaden: Springer Gabler.

The World Economic Forum. (2013) Entrepreneurial Ecosystems around the globe and company growth dynamics. Report Summary for the Annual Meeting of the New Champions.

Wade, R. (2003 [1990]) Governing the Market: Economic Theory and the Role of Government in East Asian Industrialization. Princeton: Princeton University Press.

Wallerstein, I. (1974) The Modern World-System I: Capitalist Agriculture and the Origins of the European World-Economy in the Sixteenth Century. New York: Academic Press. 